1. Park BJ, Wannemuehler KA, Marston BJ, Goven der N, Pappas PG, Chiller TM. Estimation of the current global burden of cryptococcal meningitis among persons living with HIV/AIDS. AIDS 2009;23(4):525-530

2. Casadevall A, Perfect JR. Cryptococcus neoformans. Washington, DC: American Society for Microbiology; 1998 .

3. Goldman DL, et al. Serologic evidence for Cryptococcus infection in early childhood. Pediatrics. 2001;107(5):E66.

4. Shi M, et al. Real-time imaging of trapping and urease-dependent transmigration of Cryptococcus neoformans in mouse brain. J Clin Invest. 2010; 120(5):1683-1693

5. Chretien F, Lortholary O, Kansau I, Neuville S, Gray F, Dromer F. Pathogenesis of cerebral Cryptococcus neoformans infection after fungemia. J Infect Dis. 2002;186(4):522-530.

6. Feldmesser M, Kress Y, Novikoff P, Casadevall A. Cryptococcus neoformans is a facultative intracellular pathogen in murine pulmonary infection. Infect Immun. 2000;68(7):4225-4237.

7. Shea JM, Kechichian TB, Luberto C, Del Poeta M The cryptococcal enzyme inositol phosphosphin- golipid-phospholipase $\mathrm{C}$ confers resistance to the antifungal effects of macrophages and promotes fungal dissemination to the central nervous system. Infect Immun. 2006;74(10):5977-5988.

8. Kechichian TB, Shea J, Del PM. Depletion of alveolar macrophages decreases the dissemination of a glucosylceramide-deficient mutant of Cryptococcus neoformans in immunodeficient mice. Infect Immun. 2007;75(10):4792-4798.

9. Santangelo R, et al. Role of extracellular phospholipases and mononuclear phagocytes in dissemination of cryptococcosis in a murine model. Infect Immun. 2004;72(4):2229-2239.

10. Charlier C, Nielsen K, Daou S, Brigitte M, Chretien $\mathrm{F}$, Dromer F. Evidence of a role for monocytes in dissemination and brain invasion by Cryptococcus neoformans. Infect Immun. 2009;77(1):120-127.

11. Chang YC, et al. Cryptococcal yeast cells invade the central nervous system via transcellular penetration of the blood-brain barrier. Infect Immun. 2004;72(9):4985-4995

12. Olszewski MA, et al. Urease expression by Cryptococcus neoformans promotes microvascular sequestration, thereby enhancing central nervous system invasion. Am J Pathol. 2004;164(5):1761-1771.
13. Jong A, et al. Involvement of human CD44 during Cryptococcus neoformans infection of brain microvascular endothelial cells. Cell Microbiol. 2008;10(6):1313-1326.

14. Jong A, et al. Invasion of Cryptococcus neoformans into human brain microvascular endothelial cells requires protein kinase $\mathrm{C}$-alpha activation. Cell Microbiol. 2008;10(9):1854-1865.

15. Shao X, Mednick A, Alvarez M, van Rooijen N, Casadevall A, Goldman DL. An innate immune system cell is a major determinant of species-related susceptibility differences to fungal pneumonia. Immunol. 2005;175(5):3244-3251.

16. Alvarez M, Casadevall A. Phagosome fusion and extrusion, and host cell survival following Cryptococcus neoformans phagocytosis by macrophages. Curr Biol. 2006;16(21):2161-2165.

17. Ma H, Croudace JE, Lammas DA, May RC. Expulsion of live pathogenic yeast by macrophages. Curr Biol. 2006;16(21):2156-2160.

18. Charlier C, Chretien F, Baudrimont M, Mordelet E, Lortholary O, Dromer F. Capsule structure changes associated with Cryptococcus neoformans crossing of the blood-brain barrier. Am J Pathol. $2005 ; 166(2): 421-432$

\title{
Caveat mTOR: aberrant signaling disrupts corticogenesis
}

\author{
Lucy R. Osborne
}

Department of Medicine and Department of Molecular Genetics, University of Toronto, Toronto, Ontario, Canada.

\begin{abstract}
The mammalian target of rapamycin (mTOR) signaling pathway is activated in several disorders associated with benign tumors and malformations of the cerebral cortex. In this issue of the JCI, Orlova et al. have now definitively added another disorder to this group by demonstrating that activation of mTOR signaling is associated with polyhydramnios, megalencephaly, and symptomatic epilepsy syndrome (PMSE), which is characterized by severe intractable epilepsy and megalencephaly. PMSE is caused by lack of the pseudokinase STE20-related kinase adaptor $\alpha($ STRAD $\alpha)$, and Orlova et al. show that reduction of STRAD $\alpha$ levels during corticogenesis in the mouse results in a cellular phenotype and neuronal migration defects similar to those observed in patients with PMSE, clearly demonstrating a pivotal role for STRAD $\alpha$ in cell polarity and growth. This study helps pave the way for possible therapeutic intervention with rapamycin to control the epilepsy and learning disabilities associated with this disorder.
\end{abstract}

Development of the human cortex is a complex and finely orchestrated process. The layers of the developing neocortex are generated through neuronal migration, whereby neurons travel from their place of origin in the proliferating ventricular zone to their final position in the brain. This cortical lamination is generated from the inside out, with the neurons that contribute to each layer traveling past the existing

Conflict of interest: The author has declared that no conflict of interest exists.

Citation for this article: J Clin Invest. 2010; 120(5):1392-1395. doi:10.1172/JCI43030. layers; perturbation of this carefully controlled neuronal migration can result in heterotopia (neurons in the wrong place) and dysplasia (disorganization of the normal structure of the cortex) that disrupt the normal cortical circuitry. Imbalance between excitatory and inhibitory systems in the cortex can lead to spontaneous electrical discharge with catastrophic consequences, often in the form of intractable or medication-resistant epilepsy and severe intellectual disability (1).

Polyhydramnios, megalencephaly, and symptomatic epilepsy syndrome (PMSE), recently described in an Old Order Men- nonite population, is associated with craniofacial dysmorphism (large forehead, widely spaced eyes, and large mouth), an abnormally large brain, and severe, earlyonset intractable epilepsy (2). PMSE in this kindred is caused by homozygous deletion of a portion of the STE20-related kinase adaptor $\alpha$ gene (STRADA; encoding STRAD $\alpha$ ) on human chromosome 17 (2). STRAD $\alpha$ normally binds and exports the protein kinase serine/threonine kinase 11 (STK11; also known as LKB1) out of the nucleus, where they bind to MO25 to form a trimeric complex that has an inhibitory effect on mammalian target of rapamycin (mTOR) signaling through the sequential phosphorylation of AMPK and the tuberous sclerosis complex 1 / tuberous sclerosis complex 2 (TSC1/TSC2) complex (Figure 1 and ref. 3). Aberrant activation of the mTOR pathway was identified in the brain of an individual with PMSE by the presence of high levels of phosphorylated ribosomal S6 protein (2), a downstream target of mTOR. This finding is suggestive of a link between the cellular mechanism underlying this disorder and that previously shown to be responsible for a group of syndromes characterized by hamartomas, benign tumors composed of differ- 


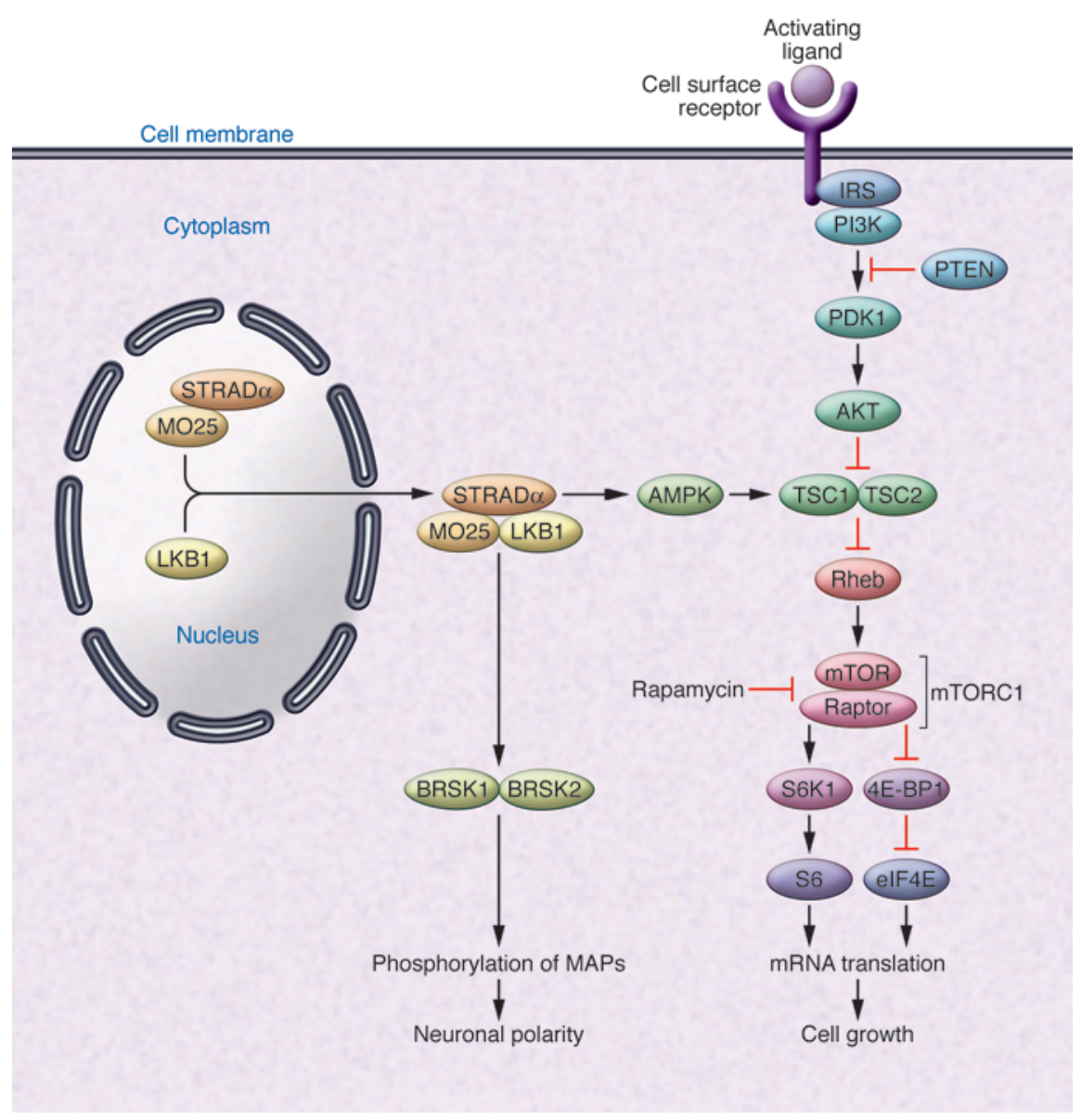

entiated but disorganized cells that form in the tissue of their origin. In this issue of the JCI, Orlova et al. have now definitively linked PMSE with the mTOR signaling pathway and elucidated the cellular mechanism by which constitutive activation of the pathway occurs (4). Specifically, they found that loss of STRAD $\alpha$ leads to activation of signaling by the mTOR complex 1 (mTORC1), a multiprotein complex that contains $\mathrm{mTOR}$ and regulatory associated protein of $\mathrm{mTOR}$ (raptor), because the trimeric STRAD $\alpha /$ LKB1/MO25 complex no longer exerts its inhibitory effects on this pathway (Figure 1).

\section{PMSE: a nonclassic hamartoma syndrome}

Regulation of mTOR signaling is essential for controlled cellular growth and proliferation, since mTOR directly phosphorylates and thereby activates important translation initiation factors, acting as a master controller of protein synthesis (5). Numerous cancers exhibit aberrant mTOR signaling, but germline mutations of the genes encoding key upstream mTOR regulators are associated with syndromes characterized by cellular overgrowth, usually in the form of hamartomas (6). Proteins that regulate the mTOR pathway therefore act as tumor suppressors, and germline mutations predispose to loss of heterozygosity and hamartoma formation (Table 1).

Because the genes that are mutated in hamartoma syndromes are functioning, at least in part, as classic tumor suppressors, we can presume that the pattern of benign growths in rapidly dividing epithelial tissues in which replication errors are most likely to occur reflects the loss of the remaining allele. The finding of Orlova et al. (4) that PMSE is also caused by dysregulation of mTOR signaling was, therefore, somewhat unexpected, since hamartomas are not a clinical feature of this syndrome. One explanation that Orlova et al. propose for the lack of tumors in patients with PMSE is that STRAD $\beta$, a pseudokinase closely related to STRAD $\alpha$, may also export LKB1 from the nucleus, albeit to a much lesser extent than STRAD $\alpha$. STRAD $\beta$ is expressed throughout the gastrointestinal tract, but not in the ventricular zone of the developing

\section{Figure 1}

mTOR signaling pathway is involved in genetic syndromes. Shown is the relationship among different proteins encoded by genes known to be responsible for genetic syndromes associated with activation of $\mathrm{mTORC} 1$. Activation of mTORC1 ultimately leads to increased protein synthesis, and thereby increased cell growth. Germline mutations that result in mTORC1 hyperactivation are therefore associated with syndromes characterized by cellular overgrowth, usually in the form of hamartomas. LKB1 also activates an mTORC1-independent pathway that controls neuronal polarity specification; it is therefore possible that the neuronal migration defects seen in individuals with PMSE may be due to effects on this pathway, and not to hyperactivation of mTOR signaling. This would explain the lack of neuronal migration defects in disorders associated with germline mutations that do not affect LKB1 localization or function. 4E-BP1, elF4E-binding protein 1; elF4E, eukaryotic translation initiation factor $4 \mathrm{E}$; MAP, microtubule-associate protein; PDK1, phosphoinositide-dependent protein kinase-1; Rheb, Ras homolog enriched in brain; S6K1, S6 kinase, $70 \mathrm{kDa}$, polypeptide 1.

cortex, which suggests that it may be able compensate for complete loss of STRAD $\alpha$ in certain tissues, but not in others $(7,8)$. This could explain why in PMSE, aberrant cell growth is only seen in the brain (in the form of megalencephaly) and in certain craniofacial regions, such as the mandible and forehead (where it manifests as craniofacial dysmorphisms). Conversely, as Orlova et al. point out (4), neurological symptoms are not seen in individuals with the hamartoma syndrome Peutz-Jeghers syndrome (PJS), which is caused by germline mutations in $L K B 1$. In PJS, however, 1 functional copy of $L K B 1$ remains, suggesting that a minimum threshold of LKB1 needs to be reached before clinical symptoms present. This idea is supported by the observation that heterozygous carriers of the PMSE-causing STRADA mutation show no apparent signs of the disease.

\section{Cellular effects of mTOR dysregulation}

Although the clinical presentation of PMSE differs from that of hamartoma syndromes, the characteristic cellular effects of mTOR 


\section{Table 1}

Genetic disorders involving activation of the mTOR pathway

$\begin{array}{lcl}\text { Disease } & \text { Gene } & \text { Clinical symptoms } \\ \text { TSC } & \text { TSC1 } & \text { Hamartomas in brain, kidney, skin, retina } \\ \text { TSC } & \text { TSC2 } & \text { Seizures, intellectual disability } \\ \text { Cowden disease (including } & \text { Hamartomas in skin, mucous membranes, breast, thyroid, and } \\ \quad \text { Lhermitte-Duclos syndrome) } & \text { rarely brain; macrocephaly, vascular anomalies } \\ \text { Bannayan-Riley-Ruvalcaba syndrome } & \text { PTEN } & \text { Hamartomas in gastrointestine, lipomas; macrocephaly, vacular anomalies } \\ \text { Macrocephaly/autism syndrome } & \text { PTEN } & \text { Macrocephaly, autism, developmental delay, obesity } \\ \text { PJS } & \text { LKB1 } & \text { Hamartomas in gastrointestine; hyperpigmented macules on lips, } \\ & \text { oral mucosa, and hands } \\ \text { PMSE } & \text { STRADA } & \text { Macrocephaly, severe epilepsy, developmental delay, hypotonia, } \\ & & \text { craniofacial dysmorphism }\end{array}$

signaling are still present. Orlova et al. demonstrated cytomegaly, hyperphosphorylation of ribosomal protein S6 (S6), and increased expression of c-MYC, another downstream target of the mTOR signaling cascade, in postmortem brain tissue from an individual with PMSE (4). LKB1 nuclear localization was unique to PMSE, as might be expected, and was not seen in the hamartoma syndrome tuberous sclerosis complex (TSC) or in other cortical dysplasias. These cellular effects were seen not only in the brain of the individual with PMSE, but also in cultured mouse neural progenitor cells after knockdown of Strada for only a few days, indicating that the cells are particularly sensitive to changes in mTOR signaling.

Neurological symptoms in disorders associated with activation of the mTOR pathway are common, with the exception of PJS, and are thought to be caused by malformations of the cerebral cortex. These cortical malformations manifest in the form of cortical tubers, subependymal nodules, and giant cell astrocytomas in TSC and rare brain hamartomas in Cowden disease, but macrocephaly and cytomegaly are also common (6). In addition to cytomegaly, Orlova et al. found evidence of neuronal heterotopia in PMSE by studying postmortem brain tissue from an infant with the disorder and performing MRI on other affected individuals; their findings suggest that STRAD $\alpha$ deficiency caused a defect in neuronal migration (4). The authors tested this hypothesis by knocking down Strada in the developing mouse cortex (E14) and found an observable defect in cortical lamination both 3 and 5 days later: cells expressing the Strada-specific shRNA knockdown construct failed to migrate properly within the cortex and became clustered in the ventricular zone.
A similar experiment using Lkb1 RNAi knockdown had previously produced an equivalent arrest in neuronal migration, which was shown to be caused by loss of cell polarity in the transfected neurons (9). The majority of transfected neurons failed to exhibit axon specification, but a small subset that managed to migrate through the cortical plate showed inverted polarity. Conditional deletion of $L k b 1$ in pyramidal neurons, the primary excitatory cells of the cortex, also results in cell polarity defects because of the inability of LKB1 to phosphorylate, and therefore activate, BR serine/threonine kinase 1 (BRSK1) and BRSK2, which are essential for neuronal polarity specification (8). Orlova et al. demonstrated that in cells lacking STRAD $\alpha$, nuclear export of LKB1 was dramatically impaired, likely seriously affecting its catalytic ability (4). This was in contrast to TSC and other cortical dysplasia syndromes, in which LKB1 localization was not altered (4). The authors therefore hypothesize that STRAD $\alpha$ may have an LKB1-independent function that is responsible for cortical malformations in PMSE; intriguingly, recent data from C. elegans suggests that in this organism, the equivalent proteins to STRAD $\alpha$ and LKB1 (STRD-1 and PAR-4, respectively) can regulate neuronal polarity through different effectors (although unlike vertebrates, C. elegans appears to have only a single STRAD isoform; ref. 10). However, the experimental data generated from the mouse, combined with the lack of documented neuronal migration defects in the hamartoma syndromes, perhaps indicate that the neuronal migration defects seen in PMSE may actually be due to effects on the distinct cell polarity pathway downstream of LKB1, not to activation of mTOR signaling. This would explain the lack of neuronal migration defects in disorders associated with proteins that do not affect LKB1 localization or function.

\section{Potential efficacy of rapamycin in PMSE}

The downstream effects of mTOR hyperactivation can be drastically altered with rapamycin, and many clinical trials in various forms of cancer associated with aberrant mTOR activation are currently ongoing. The potential for rapamycin therapy in disorders with germline mutations activating mTOR signaling is still unknown, although in TSC, it has been shown to induce regression of astrocytomas and is currently in clinical trial as a topical treatment for cutaneous lesions $(11,12)$. Successful treatment of the neurological effects in PMSE and other mTOR signaling disorders will be far more challenging, since they occur during the development and maturation of the cortex. Studies in mouse models have shown some promise, with decreased phosphorylation of the mTOR downstream target $\mathrm{S} 6$ and some reduction in cell hypertrophy and seizure activity in a mouse model of cortical dysplasia with phosphatase and tensin homolog (Pten) deletion in a subset of glutamatergic cortical neurons (13). In a second model of TSC with Tsc1 deletion in mature astrocytes, rapamycin also improved seizure activity (14), and $T s c 2^{+/-}$mice showed improvement in learning deficits when treated (15). Here, Orlova et al. showed that the cytomegaly associated with knockdown of Strada in mouse neuronal progenitors was completely alleviated with concurrent rapamycin treatment (4). It is therefore possible that rapamycin may counteract some of the ongoing cytomegaly and changes in neuronal morphology caused by activation of the mTOR pathway (16), although whether it will be 
effective against the severe epilepsy seen in individuals with PMSE and TSC remains to be seen. In both disorders, the brain insult occurs early and may not be reversible, and in the case of PMSE, the severe seizures may be caused by a combination of neuronal migration defects and mTOR activation. In rat models of epilepsy, only continuous treatment with rapamycin could counteract seizure-induced formation of new projections from axon bundles in the hippocampal region of the brain, and existing axonal reorganization could not be reversed $(17,18)$.

\section{Future directions}

Although the present study by Orlova et al. (4) definitively adds PMSE to the growing list of disorders with aberrant activation of mTOR signaling, there are questions about the pathogenesis of PMSE that remain unanswered. The relative contributions of mTOR activation and impaired LKB1 signaling to cortical dysplasia will be key in determining potential routes for therapeutic intervention. Rapamycin treatment is one approach, but partial restoration of LKB1 function may also prove helpful. A Strada knockout mouse will be extremely helpful in further determining the pre- and postnatal role of STRAD $\alpha$ in brain development and function, and will also provide a valuable tool for the testing of therapeutic agents in PMSE.

\section{Acknowledgments}

The author's work in this area is supported by the Canadian Institutes of Health Research (MOP-86457 and MOP-77720).

Address correspondence to: Lucy R. Osborne, Department of Medicine and Department of Molecular Genetics, University of Toronto, 7360 Medical Science Building, 1 King's College Circle, Toronto, Ontario M5S 1A8, Canada. Phone: 416.946.5804; Fax: 416.978.8765; E-mail: lucy.osborne@utoronto.ca.

1. Leventer RJ, Guerrini R, Dobyns WB. Malformations of cortical development and epilepsy. Dialogues Clin Neurosci. 2008;10(1):47-62.

2. Puffenberger EG, et al. Polyhydramnios, megalencephaly and symptomatic epilepsy caused by a homozygous 7-kilobase deletion in LYK5. Brain. 2007;130(Pt 7):1929-1941.

3. Inoki K, Corradetti MN, Guan KL. Dysregulation of the TSC-mTOR pathway in human disease. Nat Genet. 2005;37(1):19-24.

4. Orlova KA, et al. STRAD $\alpha$ deficiency results in aberrant mTORC1 signaling during corticogenesis in humans and mice. J Clin Invest. 2010; 120(5):1591-1602.

5. Dowling RJ, Topisirovic I, Fonseca BD, Sonenberg N. Dissecting the role of mTOR: Lessons from mTOR inhibitors. Biochim Biophys Acta. 2010;1804(3):433-439.

6. Rosner M, Hanneder M, Siegel N, Valli A, Fuchs $\mathrm{C}$, Hengstschläger $\mathrm{M}$. The mTOR pathway and its role in human genetic diseases. Mutat Res. 2008;659(3):284-292.

7. Boudeau J, et al. MO25alpha/beta interact with STRADalpha/beta enhancing their ability to bind, activate and localize LKB1 in the cytoplasm. EMBOJ. 2003;22(19):5102-5114.
8. Barnes AP, et al. LKB1 and SAD kinases define a pathway required for the polarization of cortical neurons. Cell. 2007;129(3):549-563.

9. Asada N, Sanada K, Fukada Y. LKB1 regulates neuronal migration and neuronal differentiation in the developing neocortex through centrosomal positioning. J Neurosci. 2007;27(43):11769-11775.

10. Kim JS, Hung W, Narbonne P, Roy R, Zhen M. C. elegans STRADalpha and SAD cooperatively regulate neuronal polarity and synaptic organization. Development. 2010;137(1):93-102.

11. Franz DN, et al. Rapamycin causes regression of astrocytomas in tuberous sclerosis complex. Ann Neurol. 2006;59(3):490-498.

12. Koenig MK, Northrup $H$. Topical rapamycin therapy to alleviate cutaneous manifestations of tuberous sclerosis complex (TSC) and neurofibromatosis I (NF1). http://www.clinicaltrials.gov/ct2/ show/NCT01031901.

13. Ljungberg MC, Sunnen CN, Lugo JN, Anderson AE, D'Arcangelo G. Rapamycin suppresses seizures and neuronal hypertrophy in a mouse model of cortical dysplasia. Dis Model Mech. 2009;2(7-8):389-398.

14. Zeng LH, Xu L, Gutmann DH, Wong M. Rapamycin prevents epilepsy in a mouse model of tuberous sclerosis complex. Ann Neurol. 2008; 63(4):444-453.

15. Ehninger D, et al. Reversal of learning deficits in a Tsc2+/- mouse model of tuberous sclerosis. Nat Med. 2008;14(8):843-848.

16. Tavazoie SF, Alvarez VA, Ridenour DA, Kwiatkowski DJ, Sabatini BL. Regulation of neuronal morphology and function by the tumor suppressors Tsc1 and Tsc2. Nat Neurosci. 2005;8(12):1727-1734.

17. Zeng LH, Rensing NR, Wong M. The mammalian target of rapamycin signaling pathway mediates epileptogenesis in a model of temporal lobe epilepsy. J Neurosci. 2009;29(21):6964-6972.

18. Buckmaster PS, Ingram EA, Wen X. Inhibition of the mammalian target of rapamycin signaling pathway suppresses dentate granule cell axon sprouting in a rodent model of temporal lobe epilepsy. J Neurosci. 2009;29(25):8259-8269. 